\title{
Increased prevalence of attention deficit hyperactivity disorder in children with Familial Mediterranean Fever
}

\author{
E Lavi ${ }^{*}$ I Berger, E Eisenstein, Y Berkun \\ From 8th International Congress of Familial Mediterranean Fever and Systemic Autoinflammatory Diseases \\ Dresden, Germany. 30 September - 3 October 2015
}

\begin{abstract}
Introduction
Attention deficit hyperactivity disorder (ADHD) is a developmental neuropsychiatric disorder characterized by inappropriate levels of inattention, impulsivity and hyperactivity. The cause of ADHD is unknown, but may involve both genetic and environmental factors. It has been suggested that exposure to inflammation in infancy may increase the risk for ADHD in later life. Familial Mediterranean Fever (FMF) is the most common inherited autoinflammatory disorder. In many FMF patients the inflammation persists in attack-free periods. The prevalence of ADHD among FMF patients has not been studied previously.
\end{abstract}

\section{Objectives}

To explore the prevalence of ADHD among FMF patients and to examine the relationship between FMF characteristics and ADHD.

\section{Patients and methods}

The cohort consisted of 103 consecutive children with FMF, followed in a single referral center. Clinical manifestations, demographic and genetic data were abstracted from the patients' medical records, supplemented by information obtained by interviews conducted during routine follow up visits. The presence of ADHD was assessed using the Diagnostic and Statistical Manual of Mental Disorders questionnaire (4th ed.; DSM-IV).

\section{Results}

ADHD was diagnosed in 33 (32.4\%) FMF patients, a rate significantly higher that known in our local unselected population (about 8\%). The distribution of ADHD subtypes

Hadassah-Hebrew University Medical Center, Mount Scopus, Israel, pediatrics, Jerusalem, Israel 